\title{
A Parameter for Ramanujan's Function $\chi(q)$ : Its Explicit Values and Applications
}

\author{
Nipen Saikia \\ Department of Mathematics, Rajiv Gandhi University, Rono Hills, Doimukh 791112, India \\ Correspondence should be addressed to Nipen Saikia,nipennak@yahoo.com
}

Received 3 May 2012; Accepted 28 June 2012

Academic Editors: L. Hajdu, L. S. Heath, and H. J. Ruskin

Copyright ( $) 2012$ Nipen Saikia. This is an open access article distributed under the Creative Commons Attribution License, which permits unrestricted use, distribution, and reproduction in any medium, provided the original work is properly cited.

We define a new parameter $I_{k, n}$ involving quotient of Ramanujan's function $\chi(q)$ for positive real numbers $k$ and $n$ and study its several properties. We prove some general theorems for the explicit evaluations of the parameter $I_{k, n}$ and find many explicit values. Some values of $I_{k, n}$ are then used to find some new and known values of Ramanujan's class invariant $G_{n}$.

\section{Introduction}

In Chapter 16 of his second notebook [1], Ramanujan develops the theory of theta-function. Ramanujan's general theta-function is defined by

$$
f(a, b)=\sum_{n=-\infty}^{\infty} a^{n(n+1) / 2} b^{n(n-1) / 2}, \quad|a b|<1 .
$$

After Ramanujan, for $|q|<1$, we define

$$
f(-q):=f\left(-q,-q^{2}\right)=\sum_{n=-\infty}^{\infty}(-1)^{n} q^{n(3 n-1) / 2}=(q ; q)_{\infty}
$$

where $(a ; q)_{\infty}:=\prod_{n=0}^{\infty}\left(1-a q^{n}\right)$. If $q=e^{2 \pi i z}$ with $\operatorname{Im}(z)>0$, then $f(-q)=q^{-1 / 24} \eta(z)$, where $\eta(z)$ denotes the classical Dedekind eta function.

Ramanujan's function $\chi(q)$ is defined by

$$
\chi(q):=\frac{f(q)}{f\left(-q^{2}\right)}=\left(-q ; q^{2}\right)_{\infty} .
$$

The function $\chi(q)$ is intimately connected to Ramanujan's class invariants $G_{n}$ and $g_{n}$, which are defined by

$$
G_{n}=2^{-1 / 4} q^{-1 / 24} \chi(q), \quad g_{n}=2^{-1 / 4} q^{-1 / 24} \chi(-q),
$$

where $q:=e^{-\pi \sqrt{n}}$ and $n$ is a positive rational number. Since from [2, page 124, Entry 12(v) \& (vi)]

$$
\begin{gathered}
\chi(q)=2^{1 / 6}\left\{\frac{\alpha(1-\alpha)}{q}\right\}^{-1 / 24}, \\
\chi(-q)=2^{1 / 6}(1-\alpha)^{1 / 12}\left(\frac{\alpha}{q}\right)^{-1 / 24}
\end{gathered}
$$

it follows from (4) that

$$
\begin{gathered}
G_{n}=\{4 \alpha(1-\alpha)\}^{-1 / 24}, \\
g_{n}=2^{-1 / 12}(1-\alpha)^{1 / 12} \alpha^{-1 / 24} .
\end{gathered}
$$

Also, if $\beta$ has degree $r$ over $\alpha$, then

$$
G_{r^{2} n}=\{4 \beta(1-\beta)\}^{-1 / 24}, \quad g_{r^{2} n}=2^{-1 / 12}(1-\beta)^{1 / 12} \beta^{-1 / 24} .
$$

In his notebooks [1] and paper [3], Ramanujan recorded a total of 116 class invariants or monic polynomials satisfied by them. The table at the end of Weber's book [4, page 721726] also contains the values of 107 class invariants. Weber primarily was motivated to calculate class invariants so that he could construct Hilbert class fields. On the other hand Ramanujan calculated class invariants to approximate $\pi$ and probably for finding explicit values of Rogers-Ramanujan continued fractions, theta-functions, and so on. An account 
of Ramanujan's class invariants and applications can be found in Berndt's book [5]. For further references, see [612].

Ramanujan and Weber independently and many others in the literature calculated class invariants $G_{n}$ for odd values of $n$ and $g_{n}$ for even values of $n$. For the first time, Yi [13] calculated some values of $g_{n}$ for odd values of $n$ by finding explicit values of the parameter $r_{k, n}$ (see [13, page 11, (2.1.1)] or $[14$, page $4,(1.11)])$ defined by

$$
r_{k, n}:=\frac{f(-q)}{k^{1 / 4} q^{(k-1) / 24} f\left(-q^{k}\right)}, \quad q=e^{-2 \pi \sqrt{n / k}} .
$$

In particular, she established the result [13, page 18, Theorem 2.2.3]

$$
g_{n}=r_{2, n / 2}
$$

However, the values of $G_{n}$ for even values of $n$ have not been calculated. The main objective of this paper is to evaluate some new values of $G_{n}$ for even values of $n$. We also prove some known values of $G_{n}$. For evaluation of class invariant $G_{n}$ in this paper, we introduce the parameter $I_{k, n}$, which is defined as

$$
I_{k, n}:=\frac{\chi(q)}{q^{(-k+1) / 24} \chi\left(q^{k}\right)}, \quad q=e^{-\pi \sqrt{n / k}},
$$

where $k$ and $n$ are positive real numbers.

In Section 3, we study some properties of $I_{k, n}$ and also establish its relations with Ramanujan's class invariant $G_{n}$. In Section 4, by employing Ramanujan's modular equations, we present some general theorems for the explicit evaluations of $I_{k, n}$ and find several explicit values of $I_{k, n}$. In Section 5, we establish some general theorems connecting the parameter $I_{k, n}$ and the class invariant $G_{n}$. We also evaluate some explicit values of the product $G_{n k} G_{n / k}$ by employing some values of $I_{k, n}$ evaluated in Section 4. Finally, in Section 6, we calculate new and known values of class invariant $G_{n}$ by combining the explicit values of $I_{k, n}$ and the product $G_{n k} G_{n / k}$ evaluated in Sections 4 and 5, respectively. Section 2 is devoted to record some preliminary results.

Since Ramanujan's modular equations are key in our evaluations of $I_{k, n}$ and $G_{n}$, we complete this introduction by defining Ramanujan's modular equation from Berndt's book [2]. The complete elliptic integral of the first kind $K(k)$ is defined by

$$
\begin{aligned}
K(k): & =\int_{0}^{\pi / 2} \frac{d \phi}{\sqrt{1-k^{2} \sin ^{2} \phi}}=\frac{\pi}{2} \sum_{n=0}^{\infty} \frac{(1 / 2)_{n}^{2}}{(n !)^{2}} k^{2 n} \\
& =\frac{\pi}{2}{ }_{2} F_{1}\left(\frac{1}{2}, \frac{1}{2} ; 1 ; k^{2}\right),
\end{aligned}
$$

where $0<k<1,{ }_{2} F_{1}$ denotes the ordinary or Gaussian hypergeometric function, and

$$
(a)_{n}=a(a+1)(a+2) \cdots(a+n-1) .
$$

The number $k$ is called the modulus of $K$, and $k^{\prime}:=\sqrt{1-k^{2}}$ is called the complementary modulus. Let $K, K^{\prime}, L$, and
$L^{\prime}$ denote the complete elliptic integrals of the first kind associated with the moduli $k, k^{\prime}, l$, and $l^{\prime}$, respectively. Suppose that the equality

$$
n \frac{K^{\prime}}{K}=\frac{L^{\prime}}{L}
$$

holds for some positive integer $n$. Then, a modular equation of degree $n$ is a relation between the moduli $k$ and $l$, which is implied by (13).

If we set

$$
q=\exp \left(-\pi \frac{K^{\prime}}{K}\right), \quad q^{\prime}=\exp \left(-\pi \frac{L^{\prime}}{L}\right),
$$

we see that (13) is equivalent to the relation $q^{n}=q^{\prime}$. Thus, a modular equation can be viewed as an identity involving theta-functions at the arguments $q$ and $q^{n}$. Ramanujan recorded his modular equations in terms of $\alpha$ and $\beta$, where $\alpha=k^{2}$ and $\beta=l^{2}$. We say that $\beta$ has degree $n$ over $\alpha$. The multiplier $m$ connecting $\alpha$ and $\beta$ is defined by

$$
m=\frac{K}{L} .
$$

Ramanujan also established many "mixed" modular equations in which four distinct moduli appear, which we define from Berndt's book [2, page 325].

Let $K, K^{\prime}, L_{1}, L_{1}^{\prime}, L_{2}, L_{2}^{\prime}, L_{3}$, and $L_{3}^{\prime}$ denote complete elliptic integrals of the first kind corresponding, in pairs, to the moduli $\sqrt{\alpha}, \sqrt{\beta}, \sqrt{\gamma}$, and $\sqrt{\delta}$ and their complementary moduli, respectively. Let $n_{1}, n_{2}$, and $n_{3}$ be positive integers such that $n_{3}=n_{1} n_{2}$. Suppose that the equalities

$$
n_{1} \frac{K^{\prime}}{K}=\frac{L_{1}^{\prime}}{L_{1}}, \quad n_{2} \frac{K^{\prime}}{K}=\frac{L_{2}^{\prime}}{L_{2}}, \quad n_{3} \frac{K^{\prime}}{K}=\frac{L_{3}^{\prime}}{L_{3}}
$$

hold. Then, a "mixed" modular equation is a relation between the moduli $\sqrt{\alpha}, \sqrt{\beta}, \sqrt{\gamma}$, and $\sqrt{\delta}$ that is induced by (16). In such an instance, we say that $\beta, \gamma$, and $\delta$ are of degrees $n_{1}, n_{2}$, and $n_{3}$, respectively, over $\alpha$ or $\alpha, \beta, \gamma$, and $\delta$ have degrees $1, n_{1}, n_{2}$, and $n_{3}$, respectively. Denoting $z_{r}=\phi^{2}\left(q^{r}\right)$, where

$$
q=\exp \left(-\frac{\pi K^{\prime}}{K}\right), \quad \phi(q)=f(q, q), \quad|q|<1
$$

the multipliers $m$ and $m^{\prime}$ associated with $\alpha, \beta$, and $\gamma, \delta$, respectively, are defined by $m=z_{1} / z_{n_{1}}$ and $m^{\prime}=z_{n_{2}} / z_{n_{3}}$.

\section{Preliminary Results}

Lemma 1 (see [2, page 43, Entry 27(v)]). If $\alpha$ and $\beta$ are such that the modulus of each exponential argument is less than 1 and $\alpha \beta=\pi^{2}$, then

$$
e^{\alpha / 24} \chi\left(e^{-\alpha}\right)=e^{\beta / 24} \chi\left(e^{-\beta}\right)
$$


Lemma 2 (see [15, page 241, Lemma 2.3]). Let $X:=$ $q^{1 / 12}\left(\chi(q) / \chi\left(q^{3}\right)\right)$ and $Y:=q^{1 / 6}\left(\chi\left(q^{2}\right) / \chi\left(q^{6}\right)\right)$; then

$$
\begin{aligned}
\left(\frac{X}{Y}\right)^{12}+\left(\frac{Y}{X}\right)^{12}+\left[\left(\frac{X}{Y}\right)^{6}+\left(\frac{Y}{X}\right)^{6}\right] & \\
\times & {\left[(X Y)^{10}+(X Y)^{-10}+16\left\{(X Y)^{6}+(X Y)^{-6}\right\}\right.} \\
& \left.+71\left\{(X Y)^{2}+(X Y)^{-2}\right\}\right] \\
= & (X Y)^{12}+(X Y)^{-12}+25\left\{(X Y)^{8}+(X Y)^{-8}\right\} \\
+ & 200\left\{(X Y)^{4}+(X Y)^{-4}\right\}+550 .
\end{aligned}
$$

Lemma 3 (see [15, page 241, Lemma 2.8]). Let $X:=$ $q^{1 / 6}\left(\chi(q) / \chi\left(q^{5}\right)\right)$ and $Y:=q^{1 / 3}\left(\chi\left(q^{2}\right) / \chi\left(q^{10}\right)\right)$; then

$$
\begin{gathered}
{\left[\left(\frac{X}{Y}\right)^{3}+\left(\frac{Y}{X}\right)^{3}\right]\left[(X Y)^{5}+(X Y)^{-5}+8\left((X Y)^{3}+(X Y)^{-3}\right)\right.} \\
\left.+19\left(X Y+(X Y)^{-1}\right)\right]+\left(\frac{X}{Y}\right)^{6}+\left(\frac{Y}{X}\right)^{6} \\
=\left((X Y)^{6}+(X Y)^{-6}\right)+13\left(\left((X Y)^{4}+(X Y)^{-4}\right)\right) \\
+52\left(\left((X Y)^{2}+(X Y)^{-2}\right)\right)+82 .
\end{gathered}
$$

Lemma 4 (see [15, page 252, Lemma 2.13]). Let $X:=$ $q^{1 / 4}\left(\chi(q) / \chi\left(q^{7}\right)\right)$ and $Y:=q^{1 / 2}\left(\chi\left(q^{2}\right) / \chi\left(q^{14}\right)\right)$; then

$$
\begin{gathered}
\left(\frac{X}{Y}\right)^{12}+\left(\frac{Y}{X}\right)^{12}+16\left[\left(\frac{X}{Y}\right)^{10}+\left(\frac{Y}{X}\right)^{10}\right]\left[(X Y)^{2}+(X Y)^{-2}\right] \\
+8\left[\left(\frac{X}{Y}\right)^{8}+\left(\frac{Y}{X}\right)^{8}\right]\left[7\left\{(X Y)^{4}+(X Y)^{-4}\right\}-19\right] \\
+\left[\left(\frac{X}{Y}\right)^{6}+\left(\frac{Y}{X}\right)^{6}\right] \times\left[(X Y)^{10}+(X Y)^{-10}\right. \\
+32\left\{(X Y)^{6}+(X Y)^{-6}\right\} \\
+\left[\left(\frac{X}{Y}\right)^{4}+\left(\frac{Y}{X}\right)^{4}\right]\left[16\left\{(X Y)^{8}+(X Y)^{-8}\right\}\right. \\
\left.-288\left\{(X Y)^{4}+(X Y)^{-4}\right\}+352\right] \\
+\left[\left(\frac{X}{Y}\right)^{2}+\left(\frac{Y}{X}\right)^{2}\right]\left[296\left\{(X Y)^{2}+(X Y)^{-2}\right\}\right. \\
-256\left\{(X Y)^{6}+(X Y)^{-6}\right\} \\
\left.-8\left\{(X Y)^{10}+(X Y)^{-10}\right\}\right]+1746
\end{gathered}
$$

$$
\begin{aligned}
= & \left\{(X Y)^{12}+(X Y)^{-12}\right\}+145\left[\left\{(X Y)^{8}+(X Y)^{-8}\right\}\right] \\
& +496\left[\left\{(X Y)^{4}+(X Y)^{-4}\right\}\right] .
\end{aligned}
$$

Lemma 5 (see [2, page 231, Entry 5(xii)]). Let $P=$ $\{16 \alpha \beta(1-\alpha)(1-\beta)\}^{1 / 8}$ and $Q=(\beta(1-\beta) / \alpha(1-\alpha))^{1 / 4}$; then

$$
Q+Q^{-1}+2 \sqrt{2}\left(P-P^{-1}\right)=0,
$$

where $\beta$ has degree 3 over $\alpha$.

Lemma 6 (see [2, page 282, Entry 13(xiv)]). Let $P=$ $\{16 \alpha \beta(1-\alpha)(1-\beta)\}^{1 / 12}$ and $Q=(\beta(1-\beta) / \alpha(1-\alpha))^{1 / 8}$; then

$$
Q+Q^{-1}+2\left(P-P^{-1}\right)=0,
$$

where $\beta$ has degree 5 over $\alpha$.

Lemma 7 (see [2, page 315, Entry 13(xiv)]). Let $P=$ $\{16 \alpha \beta(1-\alpha)(1-\beta)\}^{1 / 8}$ and $Q=(\beta(1-\beta) / \alpha(1-\alpha))^{1 / 6}$; then

$$
Q+Q^{-1}+7=2 \sqrt{2}\left(P+P^{-1}\right)=0,
$$

where $\beta$ has degree 7 over $\alpha$.

Lemma 8 (see $[5$, page 378, Entry 41]). Let $P=$ $2^{1 / 6}\{\alpha \beta(1-\alpha)(1-\beta)\}^{1 / 24}$ and $Q=(\beta(1-\beta) / \alpha(1-\alpha))^{1 / 24}$; then

$$
\begin{aligned}
Q^{7}+ & Q^{-7}+13\left(Q^{5}+Q^{-5}\right)+52\left(Q^{3}+Q^{-3}\right)+78\left(Q+Q^{-1}\right) \\
& -8\left(P^{-6}+P^{6}\right)=0,
\end{aligned}
$$

where $\beta$ has degree 13 over $\alpha$.

For Lemmas 9 to 15, we set

$$
\begin{aligned}
P:=(256 \alpha \beta \gamma \delta(1-\alpha)(1-\beta)(1-\gamma)(1-\delta))^{1 / 48}, \\
Q:=\left(\frac{\alpha \delta(1-\alpha)(1-\delta)}{\beta \gamma(1-\beta)(1-\gamma)}\right)^{1 / 48}, \\
R:=\left(\frac{\gamma \delta(1-\gamma)(1-\delta)}{\alpha \beta(1-\alpha)(1-\beta)}\right)^{1 / 48}, \\
T:=\left(\frac{\beta \delta(1-\beta)(1-\delta)}{\alpha \gamma(1-\alpha)(1-\gamma)}\right)^{1 / 48} .
\end{aligned}
$$

Lemma 9 (see [5, page 381, Entry 50]). If $\alpha, \beta, \gamma$, and $\delta$ have degrees $1,5,7$, and 35 , respectively, then

$$
R^{4}+R^{-4}-\left(Q^{6}+Q^{-6}\right)+5\left(Q^{4}+Q^{-4}\right)-10\left(Q^{2}+Q^{-2}\right)+15=0 .
$$

Lemma 10 (see [5, page 381, Entry 51]). If $\alpha, \beta, \gamma$, and $\delta$ have degrees $1,13,3$, and 39 , respectively, then

$$
Q^{4}+Q^{-4}-3\left(Q^{2}+Q^{-2}\right)-\left(T^{2}+T^{-2}\right)+3=0 .
$$


Lemma 11 (see [5, page 381, Entry 52]). If $\alpha, \beta, \gamma$, and $\delta$ have degrees $1,13,5$, and 65 , respectively, then

$$
Q^{6}+Q^{-6}-5\left(Q+Q^{-1}\right)^{2}\left(T+T^{-1}\right)^{2}-\left(T^{4}+T^{-4}\right)=0 .
$$

Lemma 12 (see [16, page 277, Lemma 3.1]). If $\alpha, \beta, \gamma$, and $\delta$ have degrees $1,3,7$, and 21 , respectively, then

$$
R^{2}+R^{-2}-Q^{4}-Q^{-4}+3=0 .
$$

Lemma 13 (see [15, page 243, Theorem 2.5]). If $\alpha, \beta, \gamma$, and $\delta$ have degrees $1,2,3$, and 6 , respectively, then

$$
R^{4}+R^{-4}+P^{2}-2 P^{-2}=0 .
$$

Lemma 14 (see [15, page 248, Theorem 2.10]). If $\alpha, \beta, \gamma$ and $\delta$ have degrees 1,2, 5, and 10, respectively, then

$$
R^{6}+R^{-6}+5\left(R^{2}+R^{-2}\right)=4 P^{-4}-P^{4} .
$$

Lemma 15 (see [15, page 252, Theorem 2.12]). If $\alpha, \beta$, $\gamma$ and $\delta$ have degrees 1, 2, 7, and 14, respectively, then

$$
2 \sqrt{2}\left(T^{3}+T^{-3}\right)=4 P^{-1}-2 P+4 P^{3}-P^{5} .
$$

\section{Properties of $I_{k, n}$}

In this section, we study some properties of $I_{k, n}$. We also establish a relation connecting $I_{k, n}$ and Ramanujan's class invariants $G_{n}$.

Theorem 16. For all positive real numbers $k$ and $n$, one has

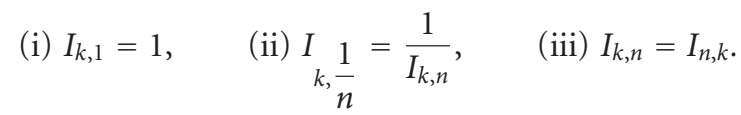

Proof. Using the definition of $I_{k, n}$ and Lemma 1, we easily arrive at (i). Replacing $n$ by $1 / n$ in $I_{k, n}$ and using Lemma 1, we find that $I_{k, n} I_{k, 1 / n}=1$, which completes the proof of (ii). To prove (iii), we use Lemma 1 in the definition of $I_{k, n}$ to arrive at $\left(I_{k, n} / I_{n, k}\right)=1$.

Remark 17. By using the definitions of $\chi(q)$ and $I_{k, n}$, it can be seen that $I_{k, n}$ has positive real value less than 1 and that the values of $I_{k, n}$ decrease as $n$ increases when $k>1$. Thus, by Theorem 16(i), $I_{k, n}<1$ for all $n>1$ if $k>1$.

Theorem 18. For all positive real numbers $k, m$, and $n$, one has

$$
I_{k, n / m}=I_{m k, n} I_{n k, m}^{-1} \text {. }
$$

Proof. Using the definition of $I_{k, n}$, we obtain

$$
\frac{I_{m k, n}}{I_{n k, m}}=\frac{\chi\left(e^{-\pi \sqrt{n / m k}}\right)}{e^{\pi(\sqrt{m / n k}-\sqrt{n / m k}) / 24} \chi\left(e^{-\pi \sqrt{m / n k}}\right)} .
$$

Using Lemma 1 in the denominator of the right-hand side of (36) and simplifying, we complete the proof.
Corollary 19. For all positive real numbers $k$ and $n$, one has

$$
I_{k^{2}, n}=I_{n k, n} I_{k, n / k}
$$

Proof. Setting $k=n$ in Theorem 18 and simplifying using Theorem 16(ii), we obtain

$$
I_{k^{2}, m}=I_{m k, k} I_{k, m / k}
$$

Replacing $m$ by $n$, we complete the proof.

Theorem 20. Let $k, a, b, c$, and $d$ be positive real numbers such that $a b=c d$. Then

$$
I_{a, b} I_{k c, k d}=I_{k a, k b} I_{c, d}
$$

Proof. From the definition of $I_{k, n}$, we deduce that, for positive real numbers $k, a, b, c$, and $d$,

$$
\begin{aligned}
I_{k a, k b} I_{a, b}^{-1} & =\frac{\chi\left(e^{-\pi \sqrt{a b}}\right)}{e^{\pi(k \sqrt{a b}-\sqrt{a b}) / 24} \chi\left(e^{-k \pi \sqrt{a b}}\right)} \\
I_{k c, k d} I_{c, d}^{-1} & =\frac{\chi\left(e^{-\pi \sqrt{c d}}\right)}{e^{\pi(k \sqrt{c d}-\sqrt{c d}) / 24} \chi\left(e^{-k \pi \sqrt{c d}}\right)} .
\end{aligned}
$$

Now the result follows readily from (40), and the hypothesis that $a b=c d$.

Corollary 21. For any positive real numbers $n$ and $p$, we have

$$
I_{n p, n p}=I_{n p^{2}, n} I_{p, p} .
$$

Proof. The result follows immediately from Theorem 20 with $a=p^{2}, b=1, c=d=p$, and $k=n$.

Now, we give some relations connecting the parameter $I_{k, n}$ and Ramanujan's class invariants $G_{n}$.

Theorem 22. Let $k$ and $n$ be any positive real numbers. Then

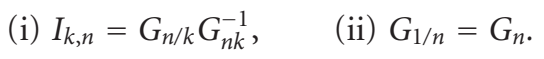

Proof. Proof of (i) follows easily from the definitions of $I_{k, n}$ and $G_{n}$ from (10) and (4), respectively. To prove (ii), we set $k=1$ in part (i) and use Theorem 16(i) and (iii).

\section{General Theorems and Explicit Evaluations of $I_{k, n}$}

In this section, we prove some general theorems for the explicit evaluations of $I_{k, n}$ and find its explicit values. 
Theorem 23. One has

$$
\begin{aligned}
& \left(\frac{I_{3, n}}{I_{3,4 n}}\right)^{12}+\left(\frac{I_{3, n}}{I_{3,4 n}}\right)^{-12}+\left[\left(\frac{I_{3, n}}{I_{3,4 n}}\right)^{6}+\left(\frac{I_{3, n}}{I_{3,4 n}}\right)^{-6}\right] \\
& \times\left[\left(I_{3, n} I_{3,4 n}\right)^{10}+\left(I_{3, n} I_{3,4 n}\right)^{-10}+16\left(\left(I_{3, n} I_{3,4 n}\right)^{6}+\left(I_{3, n} I_{3,4 n}\right)^{-6}\right)\right. \\
& \left.\quad+71\left(\left(I_{3, n} I_{3,4 n}\right)^{2}+\left(I_{3, n} I_{3,4 n}\right)^{-2}\right)\right] \\
& =\left(I_{3, n} I_{3,4 n}\right)^{12}+\left(I_{3, n} I_{3,4 n}\right)^{-12}+25\left(\left(I_{3, n} I_{3,4 n}\right)^{8}+\left(I_{3, n} I_{3,4 n}\right)^{-8}\right) \\
& +200\left(\left(I_{3, n} I_{3,4 n}\right)^{4}+\left(I_{3, n} I_{3,4 n}\right)^{-4}\right)+550 .
\end{aligned}
$$

Proof. The proof follows easily from the definition of $I_{k, n}$ and Lemma 2.

Corollary 24. One has

$$
\begin{aligned}
& \text { (i) } I_{3,2}=(-44+27 \sqrt{3}-3 \sqrt{458-264 \sqrt{3}})^{1 / 12}, \\
& \text { (ii) } I_{3,4}=\left(\frac{(\sqrt{-2+3 \sqrt{6}}-\sqrt{-6+3 \sqrt{6}})}{2}\right)^{1 / 2}, \\
& \text { (iii) } I_{3,1 / 2}=(-44+27 \sqrt{3}+3 \sqrt{458-264 \sqrt{3}})^{1 / 12} \text {, } \\
& \text { (iv) } I_{3,1 / 4}=\left(\frac{(\sqrt{-6+3 \sqrt{6}}+\sqrt{-2+3 \sqrt{6}})}{2}\right)^{1 / 2} .
\end{aligned}
$$

Proof. Setting $n=1 / 2$ in Theorem 23 and using Theorem 16(ii), we obtain

$$
I_{3,2}^{24}+I_{3,2}^{-24}+176\left(I_{3,2}^{12}+I_{3,2}^{-12}\right)-1002=0 .
$$

Equivalently,

$$
B^{2}+176 B-1004=0,
$$

where

$$
B=I_{3,2}^{12}+I_{3,2}^{-12} \text {. }
$$

Solving (46) and using the fact in Remark 17, we obtain

$$
B=54 \sqrt{5}-88 .
$$

Employing (48) in (47), solving the resulting equation for $I_{3,2}$, and noting that $I_{3,2}<1$, we arrive at

$$
I_{3,2}=(-44+27 \sqrt{3}-3 \sqrt{458-264 \sqrt{3}})^{1 / 12} .
$$

This completes the proof of (i).

Again setting $n=1$ in Theorem 23 and using Theorem 16(i), we obtain

$$
\begin{aligned}
&\left(I_{3,4}^{6}+I_{3,4}^{-6}\right)[\left(I_{3,4}^{10}+I_{3,4}^{-10}\right) \\
&\left.+16\left(I_{3,4}^{6}+I_{3,4}^{-6}\right)+71\left(I_{3,4}^{2}+I_{3,4}^{-2}\right)\right] \\
&=25\left(I_{3,4}^{8}+I_{3,4}^{-8}\right)+200\left(I_{3,4}^{4}+I_{3,4}^{-4}\right)+550 .
\end{aligned}
$$

Equivalently,

$$
\left(D^{2}+1\right)^{2}\left(D^{4}+4 D^{2}-50\right)=0
$$

where

$$
D=I_{3,4}^{2}+I_{3,4}^{-2} \text {. }
$$

Since the first factor of (51) is nonzero, solving the second factor, we deduce that

$$
D=(-2+3 \sqrt{6})^{1 / 2}
$$

Employing (53) in (52), solving the resulting equation, and using the fact that $I_{3,4}<1$, we obtain

$$
I_{3,4}=\left(\frac{(\sqrt{-2+3 \sqrt{6}}-\sqrt{-6+3 \sqrt{6}})}{2}\right)^{1 / 2} .
$$

This completes the proof of (ii).

Now (iii) and (iv) follow from (i) and (ii), respectively, and Theorem 16(ii).

Theorem 25. One has

$$
\begin{aligned}
& \left(\frac{I_{5, n}}{I_{5,4 n}}\right)^{6}+\left(\frac{I_{5, n}}{I_{5,4 n}}\right)^{-6}+\left[\left(\frac{I_{5, n}}{I_{5,4 n}}\right)^{3}+\left(\frac{I_{5, n}}{I_{5,4 n}}\right)^{-3}\right] \\
& \times\left[\left(I_{5, n} I_{5,4 n}\right)^{5}+\left(I_{5, n} I_{5,4 n}\right)^{-5}+8\left(\left(I_{5, n} I_{5,4 n}\right)^{3}+\left(I_{5, n} I_{5,4 n}\right)^{-3}\right)\right. \\
& \left.\quad+19\left(\left(I_{5, n} I_{5,4 n}\right)+\left(I_{5, n} I_{5,4 n}\right)\right)\right] \\
& =\left(I_{5, n} I_{5,4 n}\right)^{6}+\left(I_{5, n} I_{5,4 n}\right)^{-6}+13\left(\left(I_{5, n} I_{5,4 n}\right)^{4}+\left(I_{5, n} I_{5,4 n}\right)^{-4}\right) \\
& +52\left(\left(I_{5, n} I_{5,4 n}\right)^{2}+\left(I_{5, n} I_{5,4 n}\right)^{-2}\right)+82 .
\end{aligned}
$$

Proof. The proof follows from Lemma 3 and the definition of $I_{k, n}$.

Corollary 26. One has

(i) $I_{5,2}=(-14+5 \sqrt{10}-\sqrt{445-140 \sqrt{10}})^{1 / 6}$,

(ii) $I_{5,4}=\frac{\left((11+5 \sqrt{5})^{1 / 4}-\sqrt{-4+\sqrt{11+5 \sqrt{5}}}\right)}{2}$,

(iii) $I_{5,1 / 2}=(-14+5 \sqrt{10}+\sqrt{445-140 \sqrt{10}})^{1 / 6}$,

(iv) $I_{5,1 / 4}=\frac{\left((11+5 \sqrt{5})^{1 / 4}+\sqrt{-4+\sqrt{11+5 \sqrt{5}}}\right)}{2}$.

Proof. Setting $n=1 / 2$ in Theorem 25 and using Theorem 16(ii), we obtain

$$
C^{2}+56 C-216=0,
$$


where

$$
C=I_{5,2}^{6}+I_{5,2}^{-6} \text {. }
$$

Solving (57) and noting the fact in Remark 17, we obtain

$$
C=-28+10 \sqrt{10} \text {. }
$$

Employing (59) in (58), solving the resulting equation, and noting that $I_{5,2}<1$, we obtain

$$
I_{5,2}=(-14+5 \sqrt{10}-\sqrt{445-140 \sqrt{10}})^{1 / 6} .
$$

This completes the proof of (i).

Again, setting $n=1$ in Theorem 25 and using Theorem 16(i), we obtain

$$
B^{8}-22 B^{4}-4=0,
$$

where

$$
B=I_{5,4}+I_{4,5}^{-1} \text {. }
$$

Solving (61), we obtain

$$
B=(11+5 \sqrt{5})^{1 / 4}
$$

Using (63) in (62), solving the resulting equation, and noting that $I_{5,4}<1$, we arrive at

$$
I_{5,4}=\frac{\left((11+5 \sqrt{5})^{1 / 4}-\sqrt{-4+\sqrt{11+5 \sqrt{5}}}\right)}{2} .
$$

This completes the proof of (ii).

Now (iii) and (iv) follow from (i) and (ii), respectively, and Theorem 16(ii).

Theorem 27. One has

$$
\begin{aligned}
& \left(\frac{I_{7, n}}{I_{4,7 n}}\right)^{12}+\left(\frac{I_{7, n}}{I_{4,7 n}}\right)^{-12} \\
& +16\left[\left(\frac{I_{7, n}}{I_{4,7 n}}\right)^{10}+\left(\frac{I_{7, n}}{I_{4,7 n}}\right)^{-10}\right]\left[\left(I_{7, n} I_{7,4 n}\right)^{2}+\left(I_{7, n} I_{7,4 n}\right)^{-2}\right] \\
& +8\left[\left(\frac{I_{7, n}}{I_{4,7 n}}\right)^{8}+\left(\frac{I_{7, n}}{I_{4,7 n}}\right)^{-8}\right] \\
& \times\left[7\left\{\left(I_{7, n} I_{7,4 n}\right)^{4}+\left(I_{7, n} I_{7,4 n}\right)^{-4}\right\}-19\right] \\
& +\left[\left(\frac{I_{7, n}}{I_{4,7 n}}\right)^{6}+\left(\frac{I_{7, n}}{I_{4,7 n}}\right)^{-6}\right]
\end{aligned}
$$

$$
\begin{aligned}
\times & {\left[\left(I_{7, n} I_{7,4 n}\right)^{10}+\left(I_{7, n} I_{7,4 n}\right)^{-10}\right.} \\
& +32\left\{\left(I_{7, n} I_{7,4 n}\right)^{6}+\left(I_{7, n} I_{7,4 n}\right)^{-6}\right\} \\
& \left.-81\left\{\left(I_{7, n} I_{7,4 n}\right)^{2}+\left(I_{7, n} I_{7,4 n}\right)^{-2}\right\}\right] \\
+ & {\left[\left(\frac{I_{7, n}}{I_{4,7 n}}\right)^{4}+\left(\frac{I_{7, n}}{I_{4,7 n}}\right)^{-4}\right] } \\
\times & {\left[16\left\{\left(I_{7, n} I_{7,4 n}\right)^{8}+\left(I_{7, n} I_{7,4 n}\right)^{-8}\right\}\right.} \\
& \left.-288\left\{\left(I_{7, n} I_{7,4 n}\right)^{4}+\left(I_{7, n} I_{7,4 n}\right)^{-4}\right\}+352\right] \\
+ & {\left[\left(\frac{I_{7, n}}{I_{4,7 n}}\right)^{2}+\left(\frac{I_{7, n}}{I_{4,7 n}}\right)^{-2}\right] } \\
\times & {\left[296\left\{\left(I_{7, n} I_{7,4 n}\right)^{2}+\left(I_{7, n} I_{7,4 n}\right)^{-2}\right\}\right.} \\
& -256\left\{\left(I_{7, n} I_{7,4 n}\right)^{6}+\left(I_{7, n} I_{7,4 n}\right)^{-6}\right\} \\
& \left.-8\left\{\left(I_{7, n} I_{7,4 n}\right)^{10}+\left(I_{7, n} I_{7,4 n}\right)^{-10}\right\}\right]+1746 \\
= & \left\{\left(I_{7, n} I_{7,4 n}\right)^{12}+\left(I_{7, n} I_{7,4 n}\right)^{-12}\right\} \\
+ & 145\left\{\left(I_{7, n} I_{7,4 n}\right)^{8}+\left(I_{7, n} I_{7,4 n}\right)^{-8}\right\} \\
+ & 496\left\{\left(I_{7, n} I_{7,4 n}\right)^{4}+\left(I_{7, n} I_{7,4 n}\right)^{-4}\right\} .
\end{aligned}
$$

Corollary 28. One has

(i) $I_{7,2}=2^{-1 / 4}$

$$
\begin{aligned}
& \times(-8-5 \sqrt{2}+\sqrt{163+116 \sqrt{2}} \\
& \left.-\sqrt{-4+(-8-5 \sqrt{2}+\sqrt{163+116 \sqrt{2}})^{2}}\right)^{1 / 4}
\end{aligned}
$$

(ii) $I_{7,4}=\frac{\sqrt{3 \sqrt{-6+5 \sqrt{2}}-\sqrt{-58+45 \sqrt{2}}}}{\sqrt{2}}$,

(iii) $I_{7,1 / 2}=2^{-1 / 4}$,

$$
\begin{aligned}
& \times(-8-5 \sqrt{2}+\sqrt{163+116 \sqrt{2}} \\
& \left.+\sqrt{-4+(-8-5 \sqrt{2}+\sqrt{163+116 \sqrt{2}})^{2}}\right)^{1 / 4}
\end{aligned}
$$

(iv) $I_{7,1 / 4}=\frac{\sqrt{3 \sqrt{-6+5 \sqrt{2}}+\sqrt{-58+45 \sqrt{2}}}}{\sqrt{2}}$. 
Proof. Setting $n=1 / 2$ and simplifying using Theorem 16 (ii), we obtain

$$
\begin{aligned}
& \left(I_{7,2}^{24}+I_{7,2}^{-24}\right)+32\left(I_{7,2}^{20}+I_{7,2}^{-20}\right)-40\left(I_{7,2}^{16}+I_{7,2}^{-16}\right) \\
& -96\left(I_{7,2}^{12}+I_{7,2}^{-12}\right)-192\left(I_{7,2}^{8}+I_{7,2}^{-8}\right)+64\left(I_{7,2}^{4}+I_{7,2}^{-4}\right)+462=0 .
\end{aligned}
$$

Equivalently,

$$
\left(A^{2}-4\right)\left(A^{4}+32 A^{3}-42 A^{2}-128 A-191\right)=0,
$$

where

$$
A=I_{7,2}^{4}+I_{7,2}^{-4}
$$

By using the fact in Remark 17, it is seen that the first factor of (68) is nonzero, and so from the second factor, we deduce that

$$
A=-8-5 \sqrt{2}+\sqrt{163+116 \sqrt{2}} \text {. }
$$

Combining (69) and (70) and noting that $I_{7,2}<1$, we obtain

$$
\begin{aligned}
I_{7,2}=2^{-1 / 4}( & -8-5 \sqrt{2}+\sqrt{163+116 \sqrt{2}} \\
& \left.-\sqrt{-4+(-8-5 \sqrt{2}+\sqrt{163+116 \sqrt{2}})^{2}}\right)^{1 / 4} .
\end{aligned}
$$

This completes the proof of (i).

To prove (ii), setting $n=1$ and simplifying using Theorem 16(i), we arrive at

$$
E^{2}\left(E^{2}-4\right)\left(E^{4}+108 E^{2}-1134\right)=0,
$$

where

$$
E=I_{7,4}^{2}+I_{7,4}^{-2}
$$

Using the fact in Remark 17 it is seen that the first two factors of (72) are nonzero, and so solving the third factor, we obtain

$$
E=3 \sqrt{-6+5 \sqrt{2}}
$$

Combining (73) and (74) and noting that $I_{7,4}<1$, we deduce that

$$
I_{7,4}=\frac{\sqrt{3 \sqrt{-6+5 \sqrt{2}}-\sqrt{-58+45 \sqrt{2}}}}{\sqrt{2}} .
$$

So the proof of (ii) is complete.

Now (iii) and (iv) follow from (i) and (ii), respectively, and Theorem 16(ii).
Theorem 29. One has

$$
\begin{aligned}
& \left(I_{7, n} I_{7,25 n}\right)^{2}+\left(I_{7, n} I_{7,25 n}\right)^{-2}-\left\{\left(\frac{I_{7,25 n}}{I_{7, n}}\right)^{3}+\left(\frac{I_{7,25 n}}{I_{7, n}}\right)^{-3}\right\} \\
& +5\left\{\left(\frac{I_{7,25 n}}{I_{7, n}}\right)^{2}+\left(\frac{I_{7,25 n}}{I_{7, n}}\right)^{-2}\right\} \\
& -10\left\{\left(\frac{I_{7,25 n}}{I_{7, n}}\right)+\left(\frac{I_{7,25 n}}{I_{7, n}}\right)\right\}+15=0 .
\end{aligned}
$$

Proof. Using (5) in Lemma 9, we find that

$$
Q=\sqrt{\frac{q \chi\left(q^{5}\right) \chi\left(q^{7}\right)}{\chi(q) \chi\left(q^{35}\right)}}, \quad R=\sqrt{\frac{q^{3 / 2} \chi(q) \chi\left(q^{5}\right)}{\chi\left(q^{7}\right) \chi\left(q^{35}\right)}} .
$$

Setting $q=e^{-\pi \sqrt{n / 7}}$ and using the definition of $I_{k, n}$ in (77), we get

$$
Q=\left(\frac{I_{7,25 n}}{I_{7, n}}\right)^{1 / 2}, \quad R=\left(I_{7, n} I_{7,25 n}\right)^{1 / 2} .
$$

Employing (78) in (27), we complete the proof.

Corollary 30. One has

(i) $I_{7,5}=\frac{\sqrt{h-\sqrt{-36+h^{2}}}}{\sqrt{6}}$,

(ii) $I_{7,1 / 5}=\frac{\sqrt{h+\sqrt{-36+h^{2}}}}{\sqrt{6}}$,

(iii) $I_{7,25}=\frac{\left(d-\sqrt{-144+d^{2}}\right)}{12}$,

(iv) $I_{7,1 / 25}=\frac{\left(d+\sqrt{-144+d^{2}}\right)}{12}$,

where $h=5+(62-6 \sqrt{105})^{1 / 3}+(62+6 \sqrt{105})^{1 / 3}$ and $d=$ $12+2^{2 / 3}(135-15 \sqrt{21})^{1 / 3}+2^{2 / 3}(135+15 \sqrt{21})^{1 / 3}$.

Proof. Setting $n=1 / 5$ in Theorem 29 and simplifying using Theorem 16(ii), we obtain

$$
I_{7,5}^{6}+I_{7,5}^{-6}-5\left(I_{7,5}^{4}+I_{7,5}^{-4}\right)+10\left(I_{7,5}^{2}+I_{7,5}^{-2}\right)-17=0 .
$$

Equivalently,

$$
H^{3}-5 H^{2}+7 H-7=0,
$$

where

$$
H=I_{7,5}^{2}+I_{7,5}^{-2}
$$

Solving (81) and noting the fact in Remark 17, we obtain

$$
H=\frac{\left(5+(62-6 \sqrt{105})^{1 / 3}+(62+6 \sqrt{105})^{1 / 3}\right)}{3}
$$


Combining (82) and (83) and noting that $I_{7,5}<1$, we deduce that

$$
I_{7,5}=\frac{\sqrt{h-\sqrt{-36+h^{2}}}}{\sqrt{6}}
$$

where

$$
h=5+(62-6 \sqrt{105})^{1 / 3}+(62+6 \sqrt{105})^{1 / 3} .
$$

This completes the proof of (i).

Again setting $n=1$ and simplifying using Theorem 16(i), we arrive at

$$
U^{3}-6 U^{2}+7 U-3=0
$$

where

$$
U=I_{7,25}+I_{7,25}^{-1}
$$

Solving (86) and using Remark 17, we get

$$
U=\frac{\left(6+(15(9-\sqrt{21}) / 2)^{1 / 3}+(15(9+\sqrt{21}) / 2)^{1 / 3}\right)}{3} .
$$

Combining (87) and (88) and noting that $I_{7,25}<1$, we obtain

$$
I_{7,25}=\frac{\left(d-\sqrt{-144+d^{2}}\right)}{12}
$$

where

$$
d=12+2^{2 / 3}(135-15 \sqrt{21})^{1 / 3}+2^{2 / 3}(135+15 \sqrt{21})^{1 / 3} .
$$

This completes the proof of (ii). Now (ii) and (iv) easily follow from (i) and (ii), respectively, and Theorem 16(ii).

Theorem 31. One has

$$
\begin{gathered}
\left(\frac{I_{13, n}}{I_{13,9 n}}\right)^{2}+\left(\frac{I_{13, n}}{I_{13,9 n}}\right)^{-2}-3\left\{\left(\frac{I_{13, n}}{I_{13,9 n}}\right)+\left(\frac{I_{13, n}}{I_{13,9 n}}\right)^{-1}\right\} \\
-\left\{\left(I_{13, n} I_{13,9 n}\right)+\left(I_{13, n} I_{13,9 n}\right)^{-1}\right\}+3=0 .
\end{gathered}
$$

Proof. Proceeding as in the proof of Theorem 29, using (5) in Lemma 10 , setting $q:=e^{-\pi \sqrt{n / 13}}$, and using the definition of $I_{k, n}$, we arrive at

$$
Q=\left(\frac{I_{13,9 n}}{I_{13, n}}\right)^{1 / 2}, \quad T=\left(I_{13, n} I_{13,9 n}\right)^{1 / 2}
$$

Employing (92) in (28), we complete the proof.
Corollary 32. One has

$$
\begin{aligned}
& \text { (i) } I_{13,3}=\frac{(1+\sqrt{13}-\sqrt{-2+2 \sqrt{13}})}{4}, \\
& \text { (ii) } I_{13,9}=\frac{(2+\sqrt{3}-\sqrt{3+4 \sqrt{3}})}{2}, \\
& \text { (iii) } I_{13,1 / 3}=\frac{(1+\sqrt{13}+\sqrt{-2+2 \sqrt{13}})}{4}, \\
& \text { (iv) } I_{13,1 / 9}=\frac{(2+\sqrt{3}+\sqrt{3+4 \sqrt{3}})}{2} .
\end{aligned}
$$

Proof. Setting $n=1 / 3$ in Theorem 31 and simplifying using Theorem 16(ii), we obtain

$$
V^{2}-3 V-1=0
$$

where

$$
V=I_{13,3}^{2}+I_{13,3}^{-2} \text {. }
$$

Solving (94) and using Remark 17, we get

$$
V=\frac{3+\sqrt{13}}{2} .
$$

Combining (95) and (96) and noting that $I_{13,3}<1$, we obtain

$$
I_{13,3}=\frac{(1+\sqrt{13}-\sqrt{-2+2 \sqrt{13}})}{4} .
$$

So we complete the proof of (i).

Again setting $n=1$ and using Theorem 16(i), we obtain

$$
J^{2}-4 J+1=0
$$

where

$$
J=I_{13,9}+I_{13,9}^{-1} \text {. }
$$

Solving (98) and using Remark 17, we get

$$
J=2+\sqrt{3} .
$$

Combing (99) and (100) and noting that $I_{13,9}<1$, we deduce that

$$
I_{13,9}=\frac{(2+\sqrt{3}-\sqrt{3+4 \sqrt{3}})}{2} .
$$

So the proofs of (ii) is complete. Now the proof of (iii) and (iv) follow from (i) and (ii), respectively, and Theorem 16(ii).

Theorem 33. One has

$$
\begin{aligned}
& \left(\frac{I_{13,25 n}}{I_{13, n}}\right)^{3}\left(\frac{I_{13,25 n}}{I_{13, n}}\right)^{-3}-5\left\{\left(\frac{I_{13,25 n}}{I_{13, n}}\right)+\left(\frac{I_{13,25 n}}{I_{13, n}}\right)^{-1}+2\right\} \\
& \quad \times\left\{\left(I_{13,25 n} I_{13, n}\right)+\left(I_{13,25 n} I_{13, n}\right)^{-1}+2\right\} \\
& -\left\{\left(I_{13,25 n} I_{13, n}\right)^{2}+\left(I_{13,25 n} I_{13, n}\right)^{-2}\right\}=0 .
\end{aligned}
$$


Proof. Using (5) in Lemma 11, setting $q:=e^{-\pi \sqrt{n / 13}}$, and using the definition of $I_{k, n}$, we arrive at

$$
Q=\left(\frac{I_{13,25 n}}{I_{13, n}}\right)^{1 / 2}, \quad T=\left(I_{13, n} I_{13,25 n}\right)^{1 / 2} .
$$

Employing (103) in (29), we complete the proof.

Corollary 34. One has

$$
\begin{aligned}
& \text { (i) } I_{13,5}=\frac{(\sqrt{3+\sqrt{65}-\sqrt{58+6 \sqrt{65}})}}{2}, \\
& \text { (ii) } I_{13,25}=\frac{\left(c-\sqrt{-36+c^{2}}\right)}{6}, \\
& \text { (iii) } I_{13,1 / 5}=\frac{(\sqrt{3+\sqrt{65+\sqrt{58+6 \sqrt{65}}})}}{2}, \\
& \text { (iv) } I_{13,1 / 25}=\frac{\left(c+\sqrt{-36+c^{2}}\right)}{6},
\end{aligned}
$$

where $c=6+(1080-15 \sqrt{39})^{1 / 3}+(1080+15 \sqrt{39})^{1 / 3}$.

Proof. Setting $n=1 / 5$ and simplifying using Theorem 16(ii), we arrive at

$$
L^{3}-23 L-42=0,
$$

where

$$
L=I_{13,5}^{2}+I_{13,5}^{-2} .
$$

Solving (105) and using the fact in Remark 17, we obtain

$$
L=\frac{3+\sqrt{65}}{2} .
$$

Employing (107) in (106), solving the resulting equation, and noting that $I_{13,5}<1$, we obtain

$$
I_{13,5}=\frac{(\sqrt{3+\sqrt{65}-\sqrt{58+6 \sqrt{65}}})}{2} .
$$

This completes the proof of (i).

To prove (ii), setting $n=1$ and simplifying using Theorem 16(i), we arrive at

$$
A^{3}-6 A^{2}-23 A-18=0,
$$

where

$$
A=I_{13,25}+I_{13,25}^{-1} .
$$

Solving (109) and using the fact in Remark 17, we obtain

$$
A=\frac{\left(6+(1080-15 \sqrt{39})^{1 / 3}+(1080+15 \sqrt{39})^{1 / 3}\right)}{3} .
$$

Employing (111) and (110), solving the resulting equation, and noting that $I_{13,25}<1$, we obtain

$$
I_{13,25}=\frac{\left(c-\sqrt{-36+c^{2}}\right)}{6},
$$

where $c=6+(1080-15 \sqrt{39})^{1 / 3}+(1080+15 \sqrt{39})^{1 / 3}$.

This completes the proof of (ii). Now the proofs of (iii) and (iv) follow from (i) and (ii), respectively, and Theorem 16(ii).

Theorem 35. One has

$$
\left(I_{7, n} I_{7,9 n}\right)+\left(I_{7, n} I_{7,9 n}\right)^{-1}-\left\{\left(\frac{I_{7,9 n}}{I_{7, n}}\right)^{2}+\left(\frac{I_{7,9 n}}{I_{7, n}}\right)^{-2}\right\}+3=0 .
$$

Proof. Using (5) in Lemma 12, setting $q:=e^{-\pi \sqrt{n / 7}}$, and using the definition of $I_{k, n}$, we arrive at

$$
Q=\left(\frac{I_{7,9 n}}{I_{7, n}}\right)^{1 / 2}, \quad R=\left(I_{7, n} I_{7,9 n}\right)^{1 / 2} .
$$

Employing (114) in (30), we complete the proof.

Corollary 36. One has

(i) $I_{7,3}=\left(\frac{5-\sqrt{21}}{2}\right)^{1 / 4}$,

(ii) $I_{7,9}=\frac{(1+\sqrt{21}-\sqrt{6+2 \sqrt{21}})}{4}$,

(iii) $I_{7,1 / 3}=\left(\frac{5+\sqrt{21}}{2}\right)^{1 / 4}$,

(iv) $I_{7,1 / 9}=\frac{(1+\sqrt{21}+\sqrt{6+2 \sqrt{21}})}{4}$.

Proof. Setting $n=1 / 3$ and simplifying using Theorem 16(ii), we arrive at

$$
I_{7,3}^{4}+I_{7,3}^{-4}-5=0 .
$$

Solving (116) and noting the fact in Remark 17, we obtain

$$
I_{7,3}=\left(\frac{5-\sqrt{21}}{2}\right)^{1 / 4} .
$$

This completes the proof of (i).

To prove (ii), setting $n=1$ and simplifying using Theorem 16(i), we arrive at

$$
D^{2}-D-5=0,
$$

where

$$
D=I_{7,9}+I_{7,9}^{-1} .
$$


Solving (118) and using the fact in Remark 17, we obtain

$$
D=\left(\frac{1+\sqrt{21}}{2}\right) \text {. }
$$

Employing (120) in (119), solving the resulting equation, and noting that $I_{7,9}<1$, we deduce that

$$
I_{7,9}=\frac{(1+\sqrt{21}-\sqrt{6+2 \sqrt{21}})}{4} .
$$

This completes the proof of (ii). Now the proofs of (iii) and (iv) follow from (i) and (ii), respectively, and Theorem 16(ii).

\section{General Theorems and Explicit Evaluations of $G_{n k} G_{n / k}$}

In this section we evaluate some explicit values of the product $G_{n k} G_{n / k}$ by establishing some general theorems and employing the values of $I_{k, n}$ obtained in Section 4 . We recall from Theorem 22(ii) that $G_{1 / n}=G_{n}$ for ready references in this section.

Theorem 37. One has

(i) $\left(I_{3, n} I_{3,4 n}\right)^{2}+\left(I_{3, n} I_{3,4 n}\right)^{-2}+\left(G_{n / 3} G_{4 n / 3} G_{9 n / 3} G_{36 n / 3}\right)^{-1}$ $-2\left(G_{n / 3} G_{4 n / 3} G_{9 n / 3} G_{36 n / 3}\right)=0$,

(ii) $I_{3, n}^{6}+I_{3, n}^{-6}+2 \sqrt{2}\left\{\left(G_{n / 3} G_{3 n}\right)^{-3}-\left(G_{n / 3} G_{3 n}\right)^{3}\right\}=0$.

Proof. To prove (i), using (5) in Lemma 13, setting $q:=$ $e^{-\pi \sqrt{n / 3}}$, and employing the definitions of $I_{k, n}$ and $G_{n}$, we obtain

$$
\begin{gathered}
R=\sqrt{\frac{q^{1 / 4} \chi(q) \chi\left(q^{2}\right)}{\chi\left(q^{3}\right) \chi\left(q^{6}\right)}}=\left(I_{3, n} I_{3,4 n}\right)^{1 / 2}, \\
P=\left(G_{n / 3} G_{4 n / 3} G_{9 n / 3} G_{36 n / 3}\right)^{-1 / 2} .
\end{gathered}
$$

Employing (123) in (31), we complete the proof. (ii) follows similarly from Lemma 5 and the definition of $I_{k, n}$ and $G_{n}$ with $q:=e^{-\pi \sqrt{n / 3}}$.

Corollary 38. One has

(i) $G_{6} G_{3 / 2}=\sqrt{\frac{1+\sqrt{3}}{2}}$,

(ii) $G_{12} G_{4 / 3}=2^{-13 / 6}(\sqrt{6+3 \sqrt{6}}+\sqrt{-2+3 \sqrt{6}})$,

(iii) $G_{39} G_{13 / 3}=2^{1 / 6}(3+\sqrt{13})^{1 / 3}$.

Proof. Setting $n=1 / 2$ in Theorem 37(i) and simplifying using Theorem 16(ii) and the result $G_{1 / n}=G_{n}$, we obtain

$$
2\left(G_{6} G_{3 / 2}\right)^{2}-\left(G_{6} G_{3 / 2}\right)^{-2}-2=0 .
$$

Solving (125) and noting that $G_{6} G_{3 / 2}>1$, we complete the proof of (i).

To prove (ii), setting $n=1$ in Theorem 37(i); using Theorem 16(i), and noting that $G_{1 / n}=G_{n}$, we obtain

$$
I_{3,4}^{2}+I_{3,4}^{-2}+\left(G_{3}^{2} G_{4 / 3} G_{12}\right)^{-1}-2\left(G_{3}^{2} G_{4 / 3} G_{12}\right)=0 .
$$

Employing (53) in (126), solving the resulting equation, and noting that $G_{3}^{2} G_{4 / 3} G_{12}>1$, we obtain

$$
G_{3}^{2} G_{4 / 3} G_{12}=\frac{(\sqrt{6+3 \sqrt{6}}+\sqrt{-2+3 \sqrt{6}})}{4} .
$$

Using the value $G_{3}=2^{1 / 12}$ from $[5$, p. 189] in (127), we complete the proof of (ii). obtain

To prove (iii), setting $n=13$ in Theorem 37(ii), we

$$
I_{3,13}^{6}+I_{3,13}^{-6}+2 \sqrt{2}\left\{\left(G_{3 / 13} G_{39}\right)^{-2}-\left(G_{3 / 13} G_{39}\right)^{2}\right\}=0 .
$$

Cubing (96) and then employing in (128) and solving the resulting equation, we complete the proof.

Theorem 39. One has

$$
\text { (i) } \begin{aligned}
( & \left.I_{5, n} I_{5,4 n}\right)^{3}+\left(I_{5, n} I_{5,4 n}\right)^{-3}+5\left\{\left(I_{5, n} I_{5,4 n}\right)+\left(I_{5, n} I_{5,4 n}\right)^{-1}\right\} \\
= & 4\left(G_{n / 5} G_{4 n / 5} G_{25 n / 5} G_{100 n / 5}\right)^{2} \\
& -\left(G_{n / 5} G_{4 n / 5} G_{25 n / 5} G_{100 n / 5}\right)^{-2},
\end{aligned}
$$$$
\text { (ii) } I_{5, n}^{3}+I_{5, n}^{-3}+2\left\{\left(G_{n / 5} G_{5 n}\right)^{-2}-\left(G_{n / 5} G_{5 n}\right)^{2}\right\}=0 \text {. }
$$

Proof. Using (5) in Lemma 14, setting $q:=e^{-\pi \sqrt{n / 5}}$, and employing the definitions of $I_{k, n}$ and $G_{n}$, we obtain

$$
\begin{gathered}
R=\sqrt{\frac{q^{1 / 2} \chi(q) \chi\left(q^{2}\right)}{\chi\left(q^{5}\right) \chi\left(q^{10}\right)}}=\left(I_{5, n} I_{5,4 n}\right)^{1 / 2}, \\
P=\left(G_{n / 5} G_{4 n / 5} G_{25 n / 5} G_{100 n / 5}\right)^{-1 / 2} .
\end{gathered}
$$

Employing (130) in (32), we complete the proof of (i). Similarly, (ii) follows from Lemma 6 and the definition of $I_{k, n}$ and $G_{n}$ with $q:=e^{-\pi \sqrt{n / 5}}$.

Corollary 40. One has

$$
\begin{aligned}
& \text { (i) } G_{10} G_{5 / 2}=\left(\frac{3+\sqrt{10}}{2}\right)^{1 / 4}, \\
& \text { (ii) } G_{20} G_{5 / 4}=\frac{1}{2} \sqrt{-3(11+5 \sqrt{5})^{1 / 4}+(11+5 \sqrt{5})^{3 / 4}+\mathfrak{M},}
\end{aligned}
$$

where $\mathfrak{M}$ denotes $\sqrt{16+\sqrt{11+5 \sqrt{5}}(-3+\sqrt{11+5 \sqrt{5}})^{2}}$.

Proof. Setting $n=1 / 2$ in Theorem 39(i) and simplifying using Theorem 16(ii) and the result $G_{1 / n}=G_{n}$, we obtain

$$
4\left(G_{10} G_{5 / 2}\right)^{4}-\left(G_{10} G_{5 / 2}\right)^{-4}-12=0 .
$$


Solving (132) and noting that $G_{10} G_{5 / 2}>1$, we complete the proof of (i).

For proof of (ii), setting $n=1$ in Theorem 39(ii) and simplifying using Theorem $18(\mathrm{i})$ and the result $G_{1 / n}=G_{n}$, we obtain

$$
I_{5,4}^{3}+I_{5,4}^{-3}+2\left\{\left(G_{4 / 5} G_{20}\right)^{-2}-\left(G_{4 / 5} G_{20}\right)^{2}\right\}=0 .
$$

Employing the value of $I_{5,4}+I_{5,4}^{-1}$ from (63) in (133), solving the resulting equation, and noting that $G_{4 / 5} G_{20}>1$, we get

$$
G_{4 / 5} G_{20}=\frac{1}{2} \sqrt{-3(11+5 \sqrt{5})^{1 / 4}+(11+5 \sqrt{5})^{3 / 4}+\mathfrak{M}} .
$$

So the proof is complete.

Theorem 41. One has

$$
\begin{aligned}
& 2 \sqrt{2}\left\{\left(I_{2, n} I_{2,49 n}\right)^{3 / 2}+\left(I_{2, n} I_{2,49 n}\right)^{-3 / 2}\right\} \\
&=4\left(G_{n / 2} G_{4 n / 2} G_{47 n / 2} G_{196 n / 2}\right)^{1 / 2} \\
&-2\left(G_{n / 2} G_{4 n / 2} G_{47 n / 2} G_{196 n / 2}\right)^{-1 / 2} \\
&+4\left(G_{n / 2} G_{4 n / 2} G_{47 n / 2} G_{196 n / 2}\right)^{-3 / 2} \\
&-\left(G_{n / 2} G_{4 n / 2} G_{47 n / 2} G_{196 n / 2}\right)^{-5 / 2} .
\end{aligned}
$$

Proof. Using (5) in Lemma 15, setting $q:=e^{-\pi \sqrt{n / 2}}$, and employing the definitions of $I_{k, n}$ and $G_{n}$, we obtain,

$$
\begin{gathered}
R=\sqrt{\frac{q^{1 / 3} \chi(q) \chi\left(q^{7}\right)}{\chi\left(q^{2}\right) \chi\left(q^{14}\right)}}=\left(I_{2, n} I_{2,49 n}\right)^{1 / 2}, \\
P=\left(G_{n / 2} G_{4 n / 2} G_{47 n / 2} G_{196 n / 2}\right)^{-1 / 2} .
\end{gathered}
$$

Employing (136) in (33), we complete the proof.

Corollary 42. One has

$$
G_{14} G_{7 / 2}=\frac{(2+\sqrt{4+8 \sqrt{2}})}{4} .
$$

Proof. Setting $n=1 / 7$ and simplifying using Theorem 16(ii) and the result $G_{1 / n}=G_{n}$, we get

$$
\begin{aligned}
& 4\left(G_{14} G_{7 / 2}\right)^{6}-4 \sqrt{2}\left(G_{14} G_{7 / 2}\right)^{5}-2\left(G_{14} G_{7 / 2}\right)^{4} \\
& +4\left(G_{14} G_{7 / 2}\right)^{2}-1=0 .
\end{aligned}
$$

Solving (138) and noting that $G_{14} G_{7 / 2}>1$, we complete the proof.

Theorem 43. One has

$$
I_{7, n}^{4}+I_{7, n}^{-4}+7=2 \sqrt{2}\left\{\left(G_{7 / n} G_{7 n}\right)^{-3}+\left(G_{7 / n} G_{7 n}\right)^{3}\right\} .
$$

Proof. Using (5) in Lemma 7, setting $q:=e^{-\pi \sqrt{n / 7}}$, and employing definitions of $I_{k, n}$ and $G_{n}$, we arrive at

$$
\begin{gathered}
P=\left(G_{n / 7} G_{7 n}\right)^{-3}, \\
Q=\left(\frac{q^{1 / 4} \chi(q)}{\chi\left(q^{7}\right)}\right)^{4}=I_{7, n}^{4} .
\end{gathered}
$$

Using (140) in (24), we complete the proof.
Corollary 44. One has

$$
\begin{aligned}
& \text { (i) } G_{7 / 4} G_{28}=\frac{(2+\sqrt{2})}{2}, \\
& \text { (ii) } G_{7 / 3} G_{21}=2^{-1 / 6}(3+\sqrt{7})^{1 / 3}, \\
& \text { (iii) } G_{7 / 9} G_{63}=2^{1 / 6}(5+\sqrt{21})^{1 / 3} \text {. }
\end{aligned}
$$

Proof. Setting $n=4$ in Theorem 43, we get

$$
I_{7,4}^{4}+I_{7,4}^{-4}+7=2 \sqrt{2}\left\{\left(G_{7 / 4} G_{28}\right)^{3}+\left(G_{7 / 4} G_{28}\right)^{-3}\right\} .
$$

Squaring (74) and simplifying, we obtain

$$
I_{7,4}^{4}+I_{7,4}^{-4}=-56+45 \sqrt{2} .
$$

Employing (143) in (142), solving the resulting equation, and noting that $G_{7 / 4} G_{28}>1$, we complete the proof of (i).

To prove (ii), we set $n=3$ in Theorem 43, and employing (116), we obatin

$$
\left(G_{21} G_{7 / 3}\right)^{3}+\left(G_{21} G_{7 / 3}\right)^{-3}-3 \sqrt{2}=0 .
$$

Solving (144) and noting that $G_{21} G_{7 / 3}>1$, we complete the proof.

To prove (iii), setting $n=9$ in Theorem 43 , we get

$$
\left(I_{7,9}^{4}+I_{7,9}^{-4}\right)+7=2 \sqrt{2}\left\{\left(G_{7 / 9} G_{63}\right)^{3}+\left(G_{7 / 9} G_{63}\right)^{-3}\right\} .
$$

Squaring (120) twice and simplifying, we obtain

$$
I_{7,9}^{4}+I_{7,9}^{-4}=\frac{(31+7 \sqrt{21})}{2} .
$$

Employing (146) in (145), solving the resulting equation, and noting that $G_{7 / 9} G_{63}>1$, we complete the proof.

Theorem 45. One has

$$
\begin{aligned}
& \left(I_{13, n}\right)^{7}+\left(I_{13, n}\right)^{-7}+13\left\{\left(I_{13, n}\right)^{5}+\left(I_{13, n}\right)^{-5}\right\} \\
& +52\left\{\left(I_{13, n}\right)^{3}+\left(I_{13, n}\right)^{-3}\right\}+78\left\{\left(I_{13, n}\right)+\left(I_{13, n}\right)^{-1}\right\} \\
& \quad-8\left\{\left(G_{n / 13} G_{13 n}\right)^{6}-\left(G_{n} G_{13 n}\right)^{-6}\right\}=0 .
\end{aligned}
$$

Proof. Using (5) in Lemma 8, setting $q:=e^{-\pi \sqrt{n / 13}}$, and employing definitions of $I_{k, n}$ and $G_{n}$, we arrive at

$$
P=\left(G_{n / 713} G_{13 n}\right)^{-1}, \quad Q=\left(\frac{q^{1 / 2} \chi(q)}{\chi\left(q^{13}\right)}\right)^{4}=I_{13, n} .
$$

Using (148) in (25), we complete the proof.

Corollary 46. One has

$$
G_{117} G_{13 / 9}=(450+260 \sqrt{3}+\sqrt{405299+23400 \sqrt{3}})^{1 / 6} .
$$


Proof. Setting $n=9$ in Theorem 45, we get

$$
\begin{aligned}
& \left(I_{13,9}\right)^{7}+\left(I_{13,9}\right)^{-7}+13\left\{\left(I_{13,9}\right)^{5}+\left(I_{13,9}\right)^{-5}\right\} \\
& +52\left\{\left(I_{13,9}\right)^{3}+\left(I_{13,9}\right)^{-3}\right\}+78\left\{\left(I_{13,9}\right)+\left(I_{13,9}\right)^{-1}\right\} \\
& \quad-8\left\{\left(G_{9 / 13} G_{117}\right)^{6}-\left(G_{9 / 13} G_{117}\right)^{-6}\right\}=0 .
\end{aligned}
$$

Employing (100) in (150) and simplifying, we obtain

$$
\left(G_{9 / 13} G_{117}\right)^{12}-20(45+26 \sqrt{3})\left(G_{9 / 13} G_{117}\right)^{6}+1=0 .
$$

Solving the resulting equation (151) and noting that $G_{9 / 13} G_{117}>1$, we complete the proof.

\section{New Values of Class Invariant $G_{n}$}

In this section we find some new values of Ramanujan's class invariant $G_{n}$ by using explicit values of $I_{k, n}$ and $G_{n k} G_{n / k}$ evaluated in Sections 3 and 4, respectively. For ready references in this section, we recall from Theorem 22 that $I_{k, n}=G_{n / k} / G_{n k}$. We also recall from Theorem 22(ii) that $G_{1 / n}=G_{n}$.

Theorem 47. One has

(i) $G_{3 / 2}=2^{-1 / 4}(1+\sqrt{3})^{1 / 4}$

$$
\times(-44+27 \sqrt{3}-3 \sqrt{458-264 \sqrt{3}})^{1 / 24},
$$

(ii) $G_{6}=2^{-1 / 4}(1+\sqrt{3})^{1 / 4}$

$$
\times(-44+27 \sqrt{3}+3 \sqrt{458-264 \sqrt{3}})^{1 / 24} .
$$

Proof. From Corollary 24(i), we have

$$
I_{3,2}=\left(\frac{G_{3 / 2}}{G_{6}}\right)=(-44+27 \sqrt{3}-3 \sqrt{458-264 \sqrt{3}})^{1 / 12} .
$$

Also, from Corollary 38(i), we have

$$
G_{6} G_{3 / 2}=\left(\frac{1+\sqrt{3}}{2}\right)^{1 / 2}
$$

Multiplying (153) and (154) and simplifying, we complete the proof of (i). Dividing (154) by (153) and simplifying, we complete the proof of (ii).

The proofs of the Theorems $48-56$ are identical to the proof of Theorem 47. So we give the references of the required results only.
Theorem 48. One has

$$
\begin{aligned}
& \text { (i) } G_{4 / 3}=2^{-4 / 3}(\sqrt{-2+3 \sqrt{6}}-\sqrt{-6+3 \sqrt{6}})^{1 / 4} \\
& \times(\sqrt{6+3 \sqrt{6}}+\sqrt{-2+3 \sqrt{6}})^{1 / 2}, \\
& \text { (ii) } G_{12}=2^{-4 / 3}(\sqrt{6+3 \sqrt{6}}+\sqrt{-2+3 \sqrt{6}})^{1 / 2} \\
& \times(\sqrt{-2+3 \sqrt{6}}+\sqrt{-6+3 \sqrt{6}})^{1 / 4} .
\end{aligned}
$$

Proof. We use Corollary 24(ii) and Corollary 38(ii) and proceed as in Theorem 47.

Theorem 49. One has

(i) $G_{5 / 2}=2^{-1 / 8}(3+\sqrt{10})^{1 / 8}$

$$
\times(-14+5 \sqrt{10}-\sqrt{445-140 \sqrt{10}})^{1 / 12},
$$

(ii) $G_{10}=2^{-1 / 8}(3+\sqrt{10})^{1 / 8}$

$$
\times(-14+5 \sqrt{10}+\sqrt{445-140 \sqrt{10}})^{1 / 12} .
$$

Proof. We employ Corollary 26(i) and Corollary 40(i) and proceed as in Theorem 47.

Theorem 50. One has

$$
\begin{aligned}
\text { (i) } G_{5 / 4}= & \frac{1}{2}\left((11+5 \sqrt{5})^{1 / 4}-\sqrt{-4+\sqrt{11+5 \sqrt{5}}}\right)^{1 / 2} \\
& \times\left(-3(11+5 \sqrt{5})^{1 / 4}+(11+5 \sqrt{5})^{3 / 4}\right. \\
\text { (ii) } G_{20}= & \left(11+\sqrt{16+\sqrt{11+5 \sqrt{5}}(-3+\sqrt{11+5 \sqrt{5}})^{2}}\right)^{1 / 4}-\sqrt{-4+\sqrt{11+5 \sqrt{5}})^{-1 / 2}} \\
& +\left(-3(11+5 \sqrt{5})^{1 / 4}+(11+5 \sqrt{5})^{3 / 4}\right. \\
& \left(+\sqrt{\left.16+\sqrt{11+5 \sqrt{5}(-3+\sqrt{11+5 \sqrt{5}})^{2}}\right)^{1 / 4}}\right. \\
& \quad+
\end{aligned}
$$

Proof. We employ Corollary 26(ii) and Corollary 40(ii) and proceed as in Theorem 47. 
Theorem 51. One has

(i) $G_{7 / 2}=2^{-9 / 8}(2+\sqrt{4+8 \sqrt{2}})^{1 / 2}$

$$
\begin{gathered}
\times(-8-5 \sqrt{2}+\sqrt{163+116 \sqrt{2}} \\
\left.-\sqrt{-4+(-8-5 \sqrt{2}+\sqrt{163+116 \sqrt{2}})^{2}}\right)^{1 / 8},
\end{gathered}
$$

(ii) $G_{14}=2^{-9 / 8}(2+\sqrt{4+8 \sqrt{2}})^{1 / 2}$

$$
\begin{aligned}
& \times(-8-5 \sqrt{2}+\sqrt{163+116 \sqrt{2}} \\
& \left.+\sqrt{-4+(-8-5 \sqrt{2}+\sqrt{163+116 \sqrt{2}})^{2}}\right)^{1 / 8} .
\end{aligned}
$$

Proof. We employ Corollary 28(i) and Corollary 42(i) and proceed as in Theorem 47.

Theorem 52. One has

(i) $G_{7 / 4}=2^{-3 / 4}(2+\sqrt{2})^{1 / 2}(3 \sqrt{-6+5 \sqrt{2}}-\sqrt{-58+45 \sqrt{2}})^{1 / 4}$,

(ii) $G_{28}=2^{-3 / 4}(2+\sqrt{2})^{1 / 2}(3 \sqrt{-6+5 \sqrt{2}}+\sqrt{-58+45 \sqrt{2}})^{1 / 4}$.

Proof. We employ Corollary 28(ii) and Corollary 44(i) and proceed as in Theorem 47.

Theorem 53. One has

$$
\begin{aligned}
& \text { (i) } G_{7 / 3}=2^{-5 / 24}(3+\sqrt{7})^{1 / 6}(5-\sqrt{21})^{1 / 8}, \\
& \text { (ii) } G_{21}=2^{-5 / 24}(3+\sqrt{7})^{1 / 6}(5+\sqrt{21})^{1 / 8} .
\end{aligned}
$$

The values $G_{7 / 3}$ and $G_{21}$ can also be found in $[5,6]$.

Proof. We employ Corollary 36(i) and Corollary 44(ii) and proceed as in Theorem 47.

Theorem 54. One has

(i) $G_{9 / 7}=2^{-11 / 12}(5+\sqrt{21})^{1 / 6}(1+\sqrt{21}-\sqrt{6+2 \sqrt{21}})^{1 / 2}$,

(ii) $G_{63}=2^{-11 / 12}(5+\sqrt{21})^{1 / 6}(1+\sqrt{21}+\sqrt{6+2 \sqrt{21}})^{1 / 2}$.

The value $G_{63}$ can also be found in [5, page 192].

Proof. We employ Corollary 36(ii) and Corollary 44(ii) and proceed as in Theorem 47.
Theorem 55. One has

(i) $G_{13 / 3}=2^{-11 / 12}(3+\sqrt{13})^{1 / 6}(1+\sqrt{13}-\sqrt{-2+2 \sqrt{13}})^{1 / 2}$,
(ii) $G_{39}=2^{-11 / 12}(3+\sqrt{13})^{1 / 6}(1+\sqrt{13}+\sqrt{-2+2 \sqrt{13}})^{1 / 2}$.

The values $G_{13 / 3}$ and $G_{39}$ can also be found in $[5,6]$.

Proof. We use Corollary 32(i) and Corollary 38(iii) and proceed as in Theorem 47.

Theorem 56. One has

$$
\begin{aligned}
\text { (i) } G_{13 / 9}= & 2^{-1 / 2}(450+260 \sqrt{3}+\sqrt{405299+23400 \sqrt{3}})^{1 / 12} \\
& \times(2+\sqrt{3}-\sqrt{3+4 \sqrt{3}})^{1 / 2}, \\
\text { (ii) } G_{117}= & 2^{-1 / 2}(450+260 \sqrt{3}+\sqrt{405299+23400 \sqrt{3}})^{1 / 12} \\
& \times(2+\sqrt{3}+\sqrt{3+4 \sqrt{3}})^{1 / 2} .
\end{aligned}
$$

The values $G_{39}$ and $G_{117}$ can also be found in [5, page 193].

Proof. We employ Corollary 32(ii) and Corollary 46 and proceed as in Theorem 47.

\section{References}

[1] S. Ramanujan, Notebooks, vol. 1-2, Tata Institute of Fundamental Research, Bombay, India, 1957.

[2] B. C. Berndt, Ramanujan's Notebooks. Part III, Springer, New York, NY, USA, 1991.

[3] S. Ramanujan, "Modular equations and approximations to $\pi$," Quarterly Journal of Mathematics, vol. 45, pp. 350-372, 1914.

[4] H. Weber, Lehrburg Der Algebra II,, Chelsea, New York, NY, USA, 1961.

[5] B. C. Berndt, Ramanujan's Notebooks. Part V, Springer, New York, NY, USA, 1998.

[6] N. D. Baruah, "On some class invariants of Ramanujan," The Journal of the Indian Mathematical Society, vol. 68, no. 1-4, pp. 113-131, 2001.

[7] B. C. Berndt and H. H. Chan, "Some values for the RogersRamanujan continued fraction," Canadian Journal of Mathematics, vol. 47, no. 5, pp. 897-914, 1995.

[8] B. C. Berndt, H. H. Chan, S. Y. Kang, and L. C. Zhang, "A certain quotient of eta-functions found in Ramanujan's lost notebook," Pacific Journal of Mathematics, vol. 202, no. 2, pp. 267-304, 2002.

[9] B. C. Berndt, H. H. Chan, and L.-C. Zhang, "Ramanujan's class invariants with applications to the values of $q$-continued fractions and theta functions," in Special Functions, q-Series and Related Topics, M. Ismail, D. Masson, and M. Rahman, Eds., vol. 14 of Fields Institute Communications Series, pp. 37-53, American Mathematical Society, Providence, RI, USA, 1997. 
[10] B. C. Berndt, H. H. Chan, and L. C. Zhang, "Ramanujan's class invariants and cubic continued fraction," Acta Arithmetica, vol. 73, no. 1, pp. 67-85, 1995.

[11] B. C. Berndt, H. H. Chan, and L. C. Zhang, "Ramanujan's remarkable product of theta-functions," Proceedings of the Edinburgh Mathematical Society, vol. 40, no. 3, pp. 583-612, 1997.

[12] N. Saikia, "Ramanujan's modular equations and Weber-Ramanujan's class invariants $G n$ and gn," Bulletin of Mathematical Sciences, vol. 2, no. 1, pp. 205-223, 2012.

[13] J. Yi, Construction and application of modular equation [Ph.D. thesis], University of Illionis, 2001.

[14] J. Yi, "Theta-function identities and the explicity formulas for theta-function and their applications," Journal of Mathematical Analysis and Applications, vol. 292, no. 2, pp. 381-400, 2004.

[15] K. R. Vasuki and T. G. Sreeramamurthy, "Certain new Ramanujan's Schläfli-type mixed modular equations," Journal of Mathematical Analysis and Applications, vol. 309, no. 1, pp. 238-255, 2005.

[16] N. D. Baruah, "On some of Ramanujan's Schläfli-type 'mixed' modular equations," Journal of Number Theory, vol. 100, no. 2, pp. 270-294, 2003. 


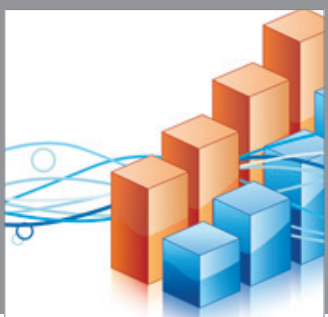

Advances in

Operations Research

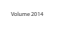

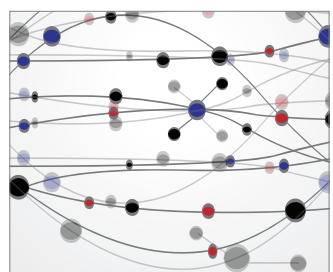

\section{The Scientific} World Journal
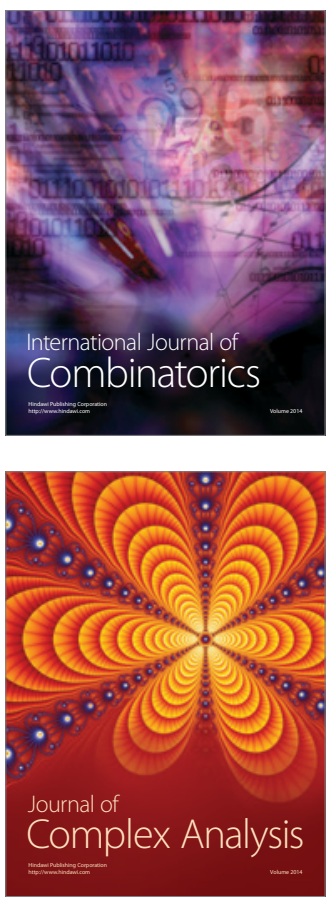

International Journal of

Mathematics and

Mathematical

Sciences
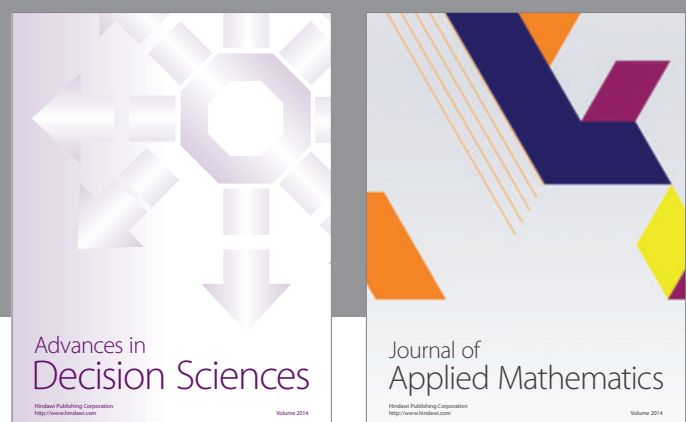

Journal of

Applied Mathematics
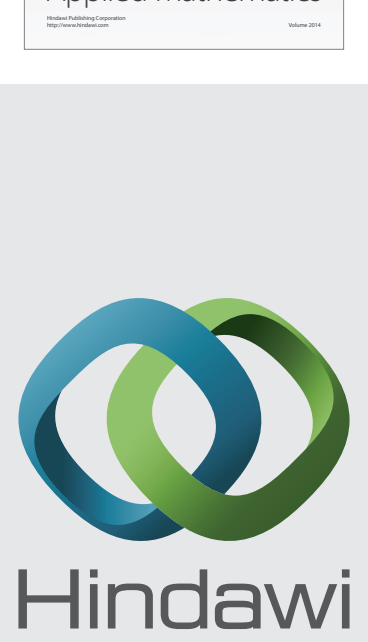

Submit your manuscripts at http://www.hindawi.com
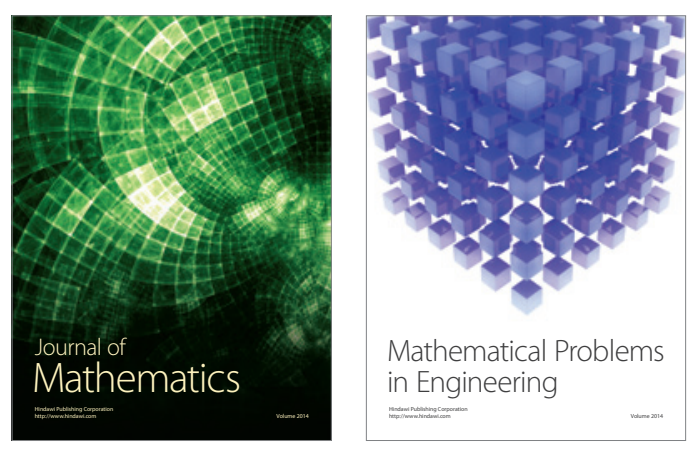

Mathematical Problems in Engineering
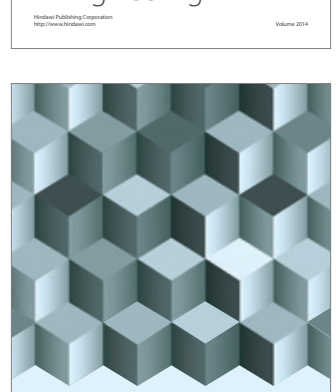

Journal of

Function Spaces
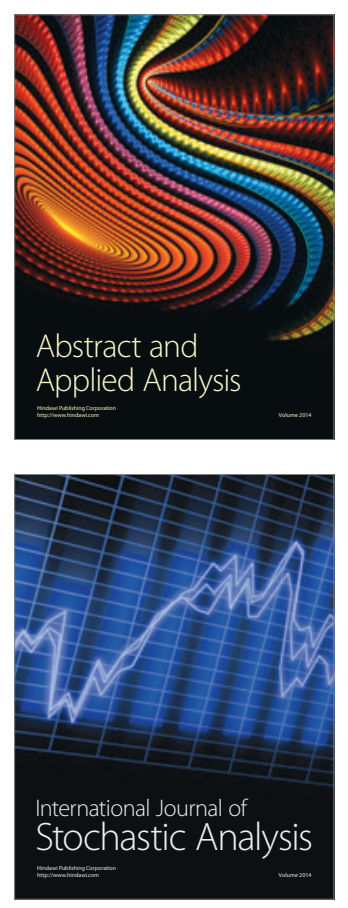

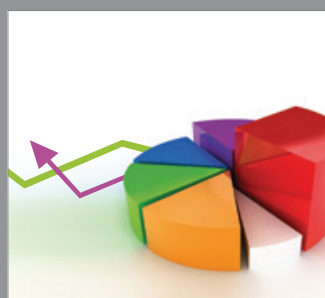

ournal of

Probability and Statistics

Promensencen
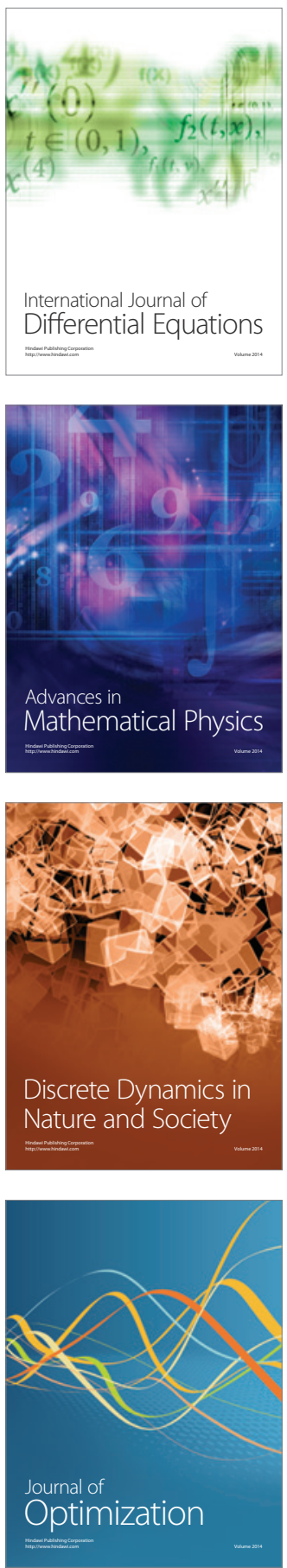